\title{
Measure the Effectiveness of an Innovative Scheme for Medical Imaging
}

\author{
Anamika Ahirwar \\ Department of Computer Science Engineering and IT \\ Maharana Pratap College of Technology \\ Gwalior (India)
}

\begin{abstract}
Automatic segmentation of tumor (cancer) region in medical imaging is an extremely challenging task. This plays a significant role in cancer research and clinical practices. The segmentation technique is widely used by the radiologists to segment the input medical image into meaningful regions. In this research, an innovative method is proposed for segmenting medical images based on SOM neural network. Then associate semantics to these regions using fuzzy reasoning. A hypothesis is established for brain MRI images and mammographs for breast cancer.

This paper divides into seven sections: First section illustrates a brief introduction about the paper. Second section describes the overview of the scheme. Third section illustrates the image pre-processing of medical diagnosis. Computation of statistical features is described in section fourth. Section fifth calculates Chi-Square values for brain MRI images and mammogram images. Scheme evaluation for brain MRI and mammogram image are described in sixth section. Finally conclude in section seven.
\end{abstract}

\section{Keywords}

Self Organizing Map(SOM), Fuzzy C-means(FCM), Cerebrospinal Fluid(CSF), Central Brain Tumor Registry of the United States(CBTRUS), The National Program of Cancer Registries(NPCR), Surveillance, Epidemiology, and End Results(SEER).

\section{INTRODUCTION}

In medical field the automated detection of the tumor region in the image are very much beneficial for the doctors and of course time saving. Automated detection of abnormal area helps doctors to give better treatment to patients. In this research two types of medical images, brain MRI and digital mammogram are worked on. Nowadays the most common type of cancer in women's is breast cancer. This is the second main cause of cancer deaths in women. If the cancer is detected at its early stages, the death rate can be reduced. At present, mammogram readings are performed by radiologists and mammographers, who visually examine mammograms for the presence of deformities that can be interpreted as cancerous changes. Manual readings may result misdiagnosis due to human errors caused by visual fatigue. To improve the diagnostic accuracy and efficiency of screening mammography, computer aided diagnosis techniques are introduced. Brain tumor is also a very critical problem among children and adults. Brain tumor is an abnormal growth of cells within the brain, which can be cancerous or noncancerous (benign). According to the report of Central Brain Tumor Registry of the United States (CBTRUS) an estimated 64,530 new cases of primary brain tumors are expected to be diagnosed in 2011 and includes both malignant $(24,070)$ and non-malignant $(40,470)$ brain tumors[9]. These estimates are based on an application of age-sex-race-specific incidence rates from the 2011 CBTRUS Statistical Report using 20042007 SEER and NPCR data1 to projected 2011 US population estimates for the respective age-sex-race groups (estimation methodology can be found at[4]. Segmentation of anatomical regions of the brain is a critical problem. In case of breast cancer, an estimated 230,480 new cases of invasive breast cancer were expected to be diagnosed in women in the U.S., along with 57,650 new cases of non-invasive breast cancer[5] in 2011. So both brain and breast cancer is serious problem nowadays.

In this paper two types of medical images, brain MRI and digital mammogram images are taking. SOM and FCM are the image processing and image analysis algorithms. So we develop an innovative scheme which is the combination of SOM and neuro fuzzy techniques[3]. To extract the semantic region of these images, integrated SOM and Fuzzy cmeans(FCM) clustering technique is applied for tumor detection in digital mammography and brain MRI images. SOM[7] or FCM[6] separately as one of the tool for the image segmentation for tumor analysis and detect the cancer (tumor) in brain MRI images and mammography images.

After extracting the regions of brain MRI and mammogram images calculate the statistical features of tumor like energy, entropy, inverse difference moment(IDM), contrast, mean and standard deviation which helps the radiologist to study the statistical information regarding tumor, so that the doctors can study the case and give better treatment to patients. For calculating these statistical properties use region growing and region merging techniques. Any computer aided analysis; the execution time is one of the important parameters for analyzing medical images. The number of tumor pixels detected. Then calculate the number of pixels affected by the tumor cells. This paper describes to measure the effectiveness of an innovative scheme for medical imaging.

Rest of the sections is describe as follows; in section II overview of the proposed scheme is described. Image preprocessing techniques for medical diagnosis are described in section III. Steps of segmenting the brain MR image to extract regions and their properties have shown in Figure1[8] and Fig 2 shows the steps of segmenting the digital mammogram image to extract tumor region and their properties. Computation of statistical features is described in section IV for both brain MRI and mammogram images. Then calculating Chi-Square values for brain MRI images and mammogram images are described in section V. Evaluation of the scheme illustrates in section VI. And finally conclude in section VII 


\section{OVERVIEW OF THE SCHEME}

We have experimented with various data mining techniques and finally designed an innovative technique using self organizing map and fuzzy reasoning for characterizing the brain MRI images and digital mammograms. An overview of the proposed scheme is shown in Fig 1. First take the input medical image (brain MRI or digital mammogram image) of size 256 by 256 . On this medical image apply the SOM and Neuro fuzzy classifier to classify the brain MRI and mammogram image.

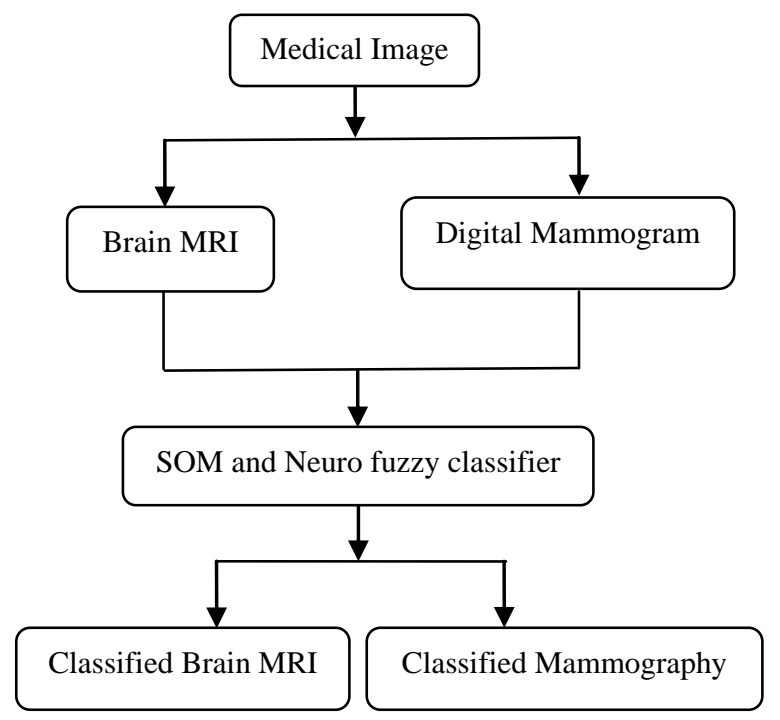

Fig 1: Overview of the scheme

\section{IMAGE PRE-PROCESSING FOR MEDICAL DIAGNOSIS}

Pre-processing is used for operations on images at the lowest level of abstraction. These iconic images are of the same kind as the original data captured by the sensor with an intensity image represented by a matrix of image function values. The aim of pre-processing is an improvement of the image data that contains undesired distortions or enhances some image features important for further processing. Image preprocessing methods use the considerable redundancy in images. Neighboring pixels corresponding to one object in real images have essentially the same or similar brightness value, so if a distorted pixel can be picked out from the image, it can usually be restored as an average value of neighboring pixels.

Image preprocessing is very important in medical diagnosis. In this work two types of images are tested i.e. brain MRI and digital mammography medical images. Using the database of brain MRI from the whole brain atlas given by Keith A. Johnson and J. Alex Becker and the database of mammograms is from the University of South Florida Digital Mammography database. The Digital Database for Screening Mammography (DDSM) is a resource for use by the mammographic image analysis research community.

Firstly, we need to do preprocessing the image which are downloaded from [1] and [2] for brain images and mammogram images respectively by using Adobe Photoshop 7.0 software. Then input a noiseless MR Image of size 256 by 256.

\subsection{MRI of Brain}

After downloading the images from the web database edit the image into 256 by 256 size, if the size varies. Then segment the image by using the integrated SOM and neuro fuzzy technique to extract the white matter, gray matter, CSF and tumor part from the image. Calculate the properties of the image (average gray scale value of region, area of region), if the image shows abnormality (tumor) then calculate the centroid of region of the image. Then further calculate the properties of tumor (energy, entropy, IDM, contrast, mean, standard deviation) of brain MRI. The steps of segmenting the brain MR image to extract region and their properties are shown in Figure1[8].

\subsection{Digital Mammogram}

The Digital Database for Screening Mammography(DDSM) is a database of digitized film-screen mammograms with associated ground truth and other information. The purpose of this resource is to provide a large set of mammograms in a digital format that may be used by researchers to evaluate and compare the performance of computer-aided detection (CAD) algorithms.

On input digital mammogram image, apply the proposed scheme to check whether the image is normal or cancerous. If the image detect tumor region then it calculate the tumor properties like centroid of the region, energy, entropy, contrast, IDM, mean and standard deviation. It also calculates the image properties like average gray scale value of the region, area of the region. Fig 2 shows the steps of segmenting the digital mammogram image to extract tumor region and their properties.

\section{COMPUTATION OF STATISTICAL FEATURES}

After preprocessing the image, my scheme[3] segments the regions of brain MRI image into white matter, gray matter, CSF and tumor region and their properties. For the computation purpose, the input features as entropy, energy, IDM, mean, contrast, standard deviation are used. Also calculate the tumor properties of mammogram image. The execution time of this technique found 45 to 60 seconds and detected tumor pixels are 588 which are approximate. Also calculate the value of type of region, average gray scale value, area of region, centroid of region (in case of segmenting tumor region), entropy, energy, IDM, mean, contrast and standard deviation of tumor pixel.

\subsection{Region Extraction of Normal and Cancerous Brain MRI Images and their Statistical Properties}

I have tested 49 axial view brain MRI images but here the result of two images are shown in Table 1 and Table 2 for normal and cancerous brain MRI image respectively. In Table 1 , input image is normal which is taken from the Keith database, but according to my scheme the result shows that it is an abnormal image. The detected pixels in area of region are less so we can consider it as a normal image. But this minor abnormality is not visible by our eyes. Table 1 shows the result of extraction of brain MRI regions and their statistical properties for normal image. Table 2 shows the result of extraction of brain MRI regions and their statistical properties for cancerous image. 


\subsection{Region Extraction of Normal and Cancerous Digital Mammogram Images and their Statistical Properties}

This scheme thus provides a feasible new solution for image segmentation of Digital Mammography for tumor detection.
Fig 3 describes the schematic diagram for detecting tumor region and their features for digital mammography image.

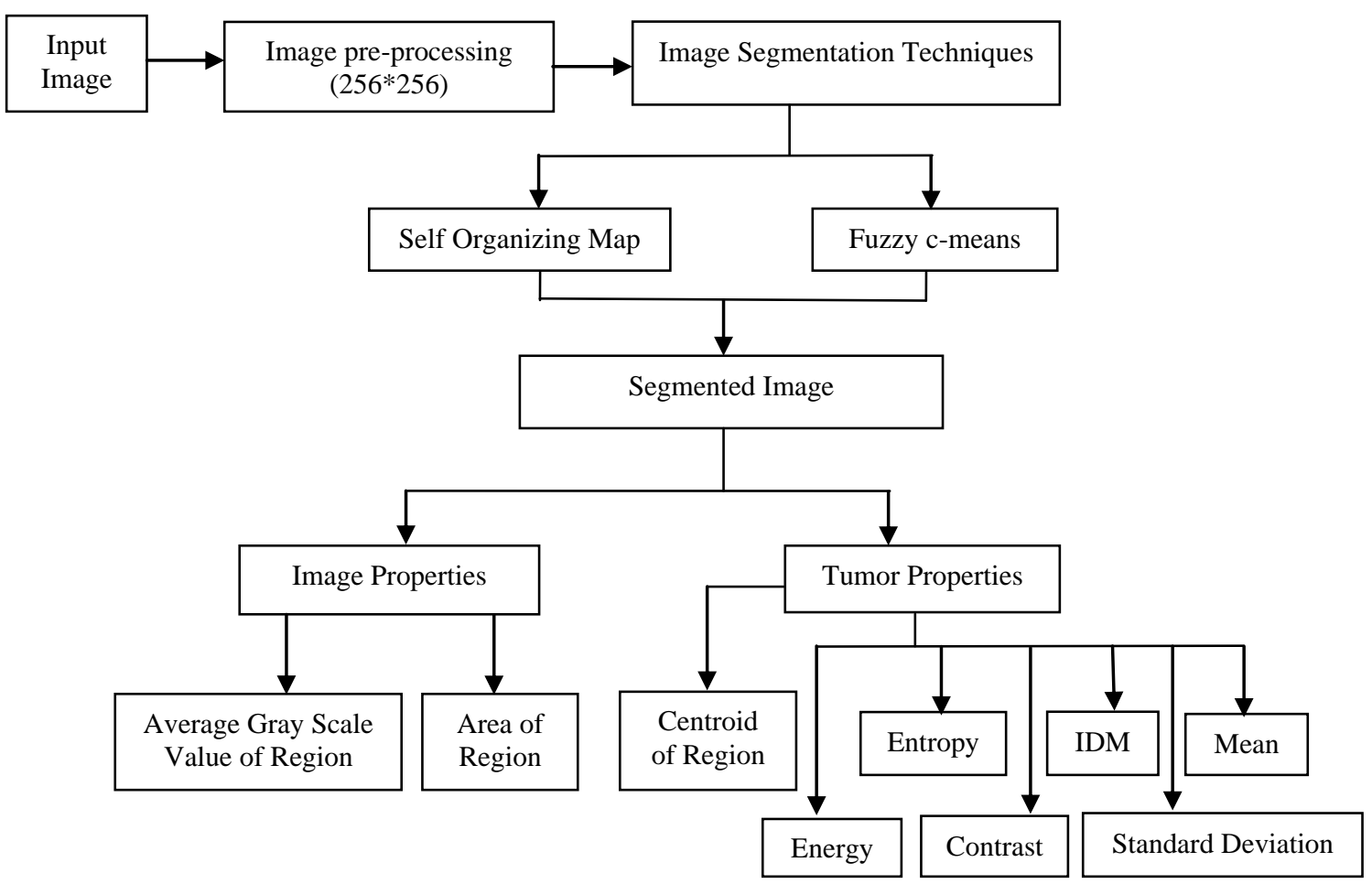

Fig 2: Steps of segmenting the digital mammogram image to extract tumor region and their properties

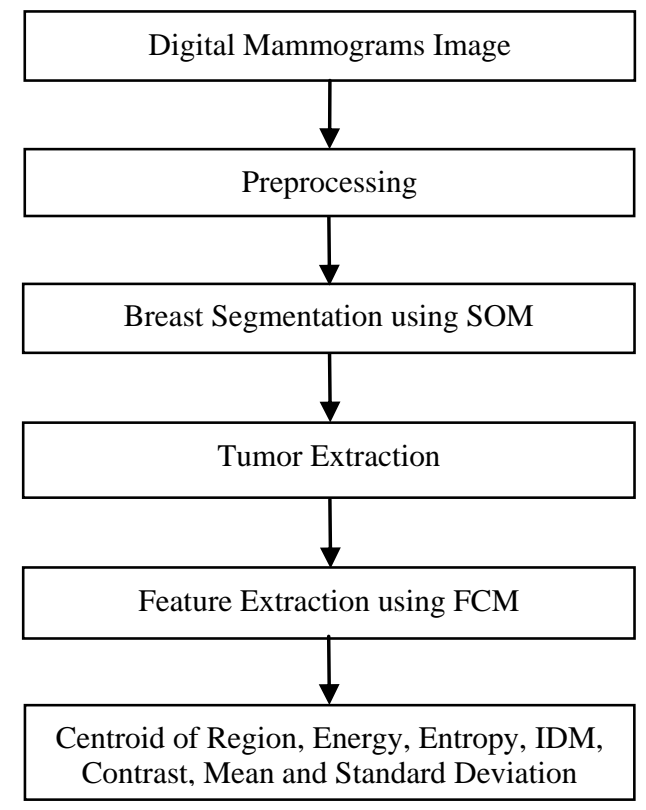

Fig 3: Schematic diagram for detecting tumor region and their features for mammography image 
Table 1: Result of extraction of brain MRI regions and their statistical properties for normal image

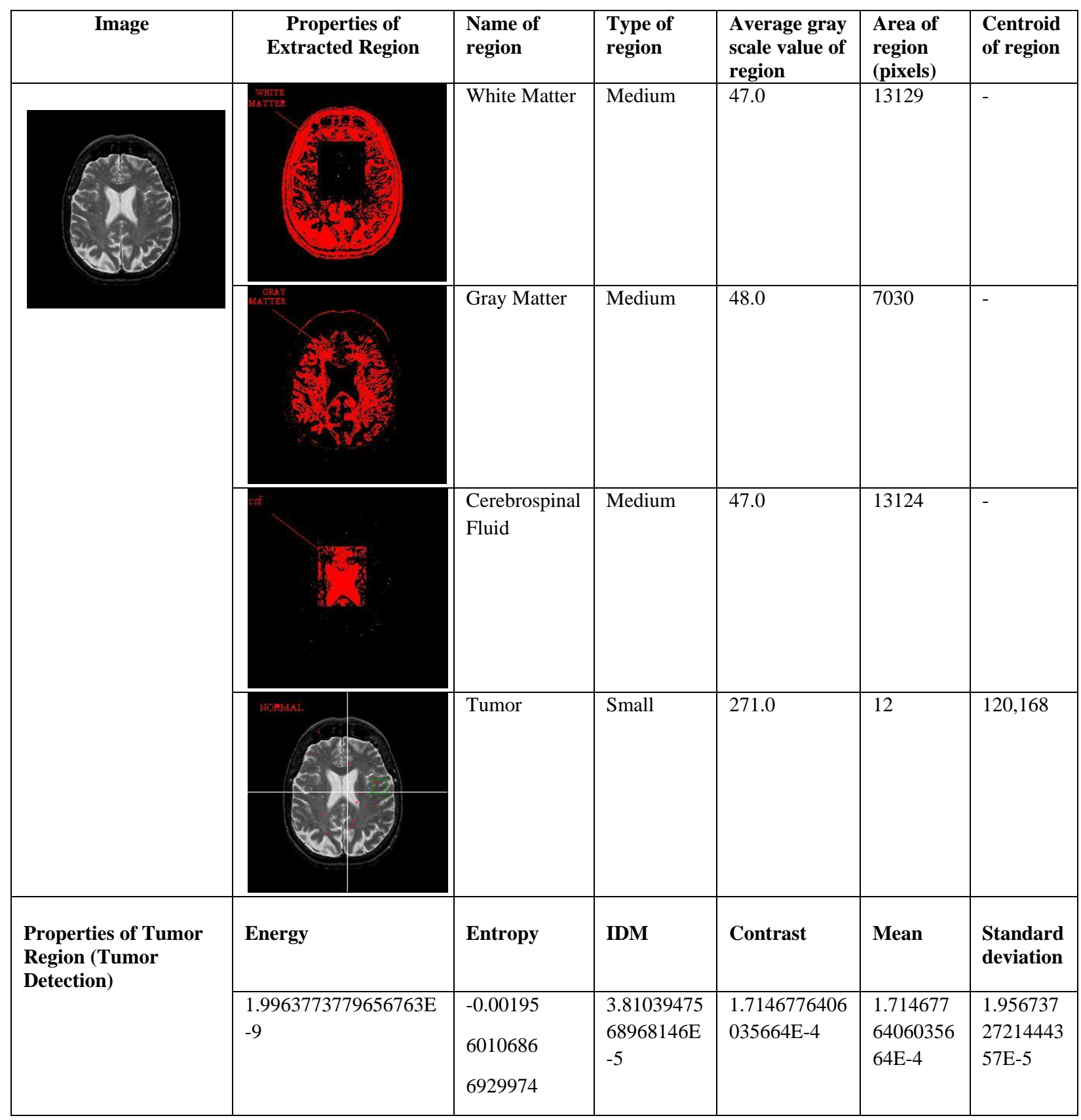


Table 2: Result of extraction of brain MRI regions and their statistical properties for cancerous image

\begin{tabular}{|c|c|c|c|c|c|c|}
\hline \multirow[t]{5}{*}{ Image } & $\begin{array}{c}\text { Properties of } \\
\text { Extracted Region }\end{array}$ & $\begin{array}{l}\text { Name of } \\
\text { region }\end{array}$ & $\begin{array}{l}\text { Type of } \\
\text { region }\end{array}$ & $\begin{array}{l}\text { Average } \\
\text { gray scale } \\
\text { value of } \\
\text { region } \\
\end{array}$ & $\begin{array}{l}\text { Area of } \\
\text { region } \\
\text { (pixels) }\end{array}$ & $\begin{array}{l}\text { Centroid } \\
\text { of region }\end{array}$ \\
\hline & WAITIE & White Matter & Medium & 33.0 & 18448 & - \\
\hline & 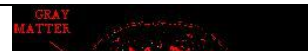 & Gray Matter & Medium & 29.0 & 10007 & - \\
\hline & & $\begin{array}{l}\text { Cerebrospinal } \\
\text { Fluid }\end{array}$ & Medium & 33.0 & 18448 & - \\
\hline & & Tumor & Small & 52.0 & 500 & 200,152 \\
\hline \multirow{2}{*}{$\begin{array}{l}\text { Properties of Tumor } \\
\text { Region (Tumor } \\
\text { Detection) }\end{array}$} & Energy & Entropy & IDM & Contrast & Mean & $\begin{array}{l}\text { Standard } \\
\text { deviation }\end{array}$ \\
\hline & $\begin{array}{l}1.1960093386154377 \mathrm{E} \\
-11\end{array}$ & $\begin{array}{l}-2.61844610 \\
84208005 \mathrm{E}-4\end{array}$ & $\begin{array}{l}2.877510 \\
1480191 \\
217 \mathrm{E}-6\end{array}$ & $\begin{array}{l}1.822423093 \\
7454446 \mathrm{E}-5\end{array}$ & $\begin{array}{l}1.822423093 \\
7454446 \mathrm{E}-5\end{array}$ & $\begin{array}{l}7.191600 \\
75772128 \\
7 \mathrm{E}-7\end{array}$ \\
\hline
\end{tabular}

I have tested 1043 mammogram images, but here I am showing the result of one normal and one cancerous mammogram image. Table 3 and Table 4 show the result of extraction of tumor region in digital mammogram and their statistical properties of normal and cancerous image respectively.

Table 3: Result of Extraction of Tumor Region in Digital Mammograms and their Statistical Properties of Normal Image

\begin{tabular}{|c|c|c|c|c|c|}
\hline Input Image & \multirow{5}{*}{ Result } & \multicolumn{2}{|c|}{ Properties of Extracted Region } & \multicolumn{2}{|c|}{$\begin{array}{c}\begin{array}{c}\text { Properties of Tumor Region } \\
\text { (Tumor Detection) }\end{array} \\
\end{array}$} \\
\hline \multirow{6}{*}{ Normal } & & Type of region & Small & Energy & $4.6100467443466187 \mathrm{E}-8$ \\
\hline & & $\begin{array}{l}\text { Average gray scale } \\
\text { value of region }\end{array}$ & 0.0 & Entropy & -0.0021438427064320734 \\
\hline & & Area of region (pixels) & -40 & IDM & $2.288818359375 \mathrm{E}-4$ \\
\hline & & Centroid of region & 8,8 & Contrast & $2.44140625 \mathrm{E}-4$ \\
\hline & Normal & & & Mean & $2.44140625 \mathrm{E}-4$ \\
\hline & & & & $\begin{array}{l}\text { Standard } \\
\text { deviation }\end{array}$ & $5.623722714419876 \mathrm{E}-6$ \\
\hline
\end{tabular}


Table 4: Result of Extraction of Tumor Region in Digital Mammograms and their Statistical Properties of cancerous image

\begin{tabular}{|c|c|c|c|c|c|}
\hline Input Image & Result & \multicolumn{2}{|c|}{ Properties of Extracted Region } & \multicolumn{2}{|c|}{$\begin{array}{l}\text { Properties of Tumor Region } \\
\text { (Tumor Detection) }\end{array}$} \\
\hline \multirow{6}{*}{ Cancer } & \multirow{6}{*}{ Cancer } & Type of region & Small & Energy & $1.4365800608474147 \mathrm{E}-11$ \\
\hline & & $\begin{array}{l}\text { Average gray scale } \\
\text { value of region }\end{array}$ & 89.0 & Entropy & $-1.4474218793470597 \mathrm{E}-4$ \\
\hline & & $\begin{array}{l}\text { Area of region } \\
\text { (pixels) }\end{array}$ & 908 & IDM & $1.0273691131749815 \mathrm{E}-5$ \\
\hline & & Centroid of region & 104,232 & Contrast & $1.0273691131749815 \mathrm{E}-5$ \\
\hline & & & & Mean & $1.0273691131749815 \mathrm{E}-5$ \\
\hline & & & & Standard deviation & $1.7466296110877375 \mathrm{E}-7$ \\
\hline
\end{tabular}

\section{CALCULATING}

CHI-SQUARE VALUES FOR BRAIN MRI IMAGES AND MAMMOGRAM IMAGES

In this section the result of tested brain MRI images and digital mammogram images are shown to calculate chi-square values. An innovative technique using self organizing map and fuzzy reasoning are applied for extracting the tumor region from the brain and mammogram images. Then compare the results from the database given on the web[1,2] and calculate the accuracy of the results of my scheme. Total 49 axial view brain MRI images and 1043 mammogram images are tested for calculating chi-square value. Table 5 and Table 6 shows calculated value of Chi-square of brain MRI images and mammogram images respectively.

Table 5: Calculating Chi-Square for brain MRI images (Axial view). Total images $=49$

\begin{tabular}{|l|l|l|}
\hline & Normal & Abnormal \\
\hline Observed (o) & 11 & 29 \\
\hline Expected (e) & 12 & 37 \\
\hline Deviation (o-e) & -1 & -8 \\
\hline Deviation $\left.\mathbf{( d}^{\mathbf{2}}\right)$ & 1 & 64 \\
\hline $\mathbf{d}^{\mathbf{2}} / \mathbf{e}$ & 0.083 & 1.73 \\
\hline $\boldsymbol{\chi} \mathbf{2}=\mathbf{d}^{\mathbf{2}} / \mathbf{e}$ & 0.083 & 1.73 \\
\hline
\end{tabular}

Table 6: Calculating Chi-Square for Mammogram images. Total images $=1043$

\begin{tabular}{|l|l|l|}
\hline & Normal & Abnormal \\
\hline Observed (o) & 444 & 551 \\
\hline Expected (e) & 444 & 609 \\
\hline Deviation (o-e) & 0 & -58 \\
\hline Deviation $\left.^{\mathbf{2}} \mathbf{d}^{\mathbf{2}}\right)$ & 0 & 3364 \\
\hline $\mathbf{d}^{2} / \mathbf{e}$ & 0 & 5.52 \\
\hline$\chi \mathbf{\chi}^{\mathbf{2}} / \mathbf{e}$ & 0 & 5.52 \\
\hline
\end{tabular}

\section{EVALUATION OF THE SCHEME}

Evaluation is very important for measuring the correctness of any new developed scheme. By evaluating this we can know how much our scheme gives accurate results. Here 49 axial view brain MRI images and 1043 mammogram images are tested from the database which is given on the web to evaluate my scheme. My scheme checks how much our result matches from the web database results. Table 7 shows the accuracy of the results of brain MRI and mammogram images.

Table 7: Accuracy of the results of brain MRI and Mammogram images

\begin{tabular}{|l|l|l|l|l|l|}
\hline \multirow{2}{*}{$\begin{array}{c}\text { Type of } \\
\text { image }\end{array}$} & $\begin{array}{c}\text { Total } \\
\text { tested } \\
\text { images }\end{array}$ & $\begin{array}{c}\text { Type of } \\
\text { image }\end{array}$ & $\begin{array}{c}\text { Total } \\
\text { images } \\
(\mathbf{e})\end{array}$ & $\begin{array}{c}\text { Total } \\
\text { images } \\
(\mathbf{o})\end{array}$ & Accuracy \\
\hline $\begin{array}{l}\text { Brain } \\
\text { MRI } \\
\text { (axial } \\
\text { view) }\end{array}$ & 49 & $\begin{array}{l}\text { Normal } \\
\text { images }\end{array}$ & 12 & 11 & $91.67 \%$ \\
\cline { 3 - 6 } & $\begin{array}{l}\text { Cancerous } \\
\text { images } \\
\text { o-gram } \\
\text { image }\end{array}$ & 1043 & 37 & 29 & $78.38 \%$ \\
\cline { 3 - 6 } & $\begin{array}{l}\text { Normal } \\
\text { images }\end{array}$ & $\begin{array}{l}\text { Cancerous } \\
\text { images }\end{array}$ & 609 & 551 & $90.47 \%$ \\
\hline
\end{tabular}

\section{CONCLUSION}

This paper describes to measure the effectiveness of the developed scheme for medical imaging. An overview of the system is described in first section. Medical imaging generally contains unknown noise and considerable uncertainty, and therefore clinically acceptable segmentation (region classification) performance is difficult to achieve. My scheme accepts only fixed size ( 256 by 256 ) input image, for this the image should be preprocessed. This approach thus provides a feasible new solution for image segmentation of brain MRI and Digital Mammography for tumor detection. In brain MRI the image is segmented into White matter, Gray matter and CSF. If there is some abnormality in the image then extract that region and calculate the statistical features of this region like energy, entropy, inverse difference moment, contrast, mean and standard deviation. This region may be tumorous region, which aims to help the radiologist to analyze the statistical information regarding brain tumor and breast cancer to give better treatment to patients. Apply all axial view images of brain MRI and mammogram images on the scheme are tested and on the basis of some statistical test calculate their chi-square values. Then compute the accuracy for brain MRI and mammogram images to measure the effectiveness of the scheme. 


\section{REFERENCES}

[1] The whole brain Atlas by Keith A. Johnson and J. Alex Becker.

[2] University of South Florida Digital Mammography Home Page.

[3] Anamika Ahirwar and R.S. Jadon, "Segmentation and Characterization of Brain MR Image Regions Using SOM and Neuro Fuzzy Techniques", published in Proceedings of the First International Conference on Emerging Trends in Soft Computing and ICT (SCIT 2011), organized by Guru Ghasidas Vishwavidyalaya, Bilaspur(C.G.) India, PP 128-131,16-17 March 2011, ISBN NO.-978-81-920913-3-4.

[4] CBTRUS Statistical Report: Primary Brain and Central Nervous System Tumors Diagnosed in the United States in 2004-2007.

[5] U.S. Breast Cancer Statistics.
[6] "An Improved Implementation of Brain Tumor Detection Using Segmentation Based on Neuro Fuzzy Technique", by S. Murugavalli and V. Rajamani, Journal of Computer Science 3 (11): 841-846, 2007, ISSN 1549-3636.

[7] Juha Vesanto and Esa Alhoniemi, "Clustering of the Self-Organizing Map". IEEE Transactions on Neural Networks, 11(2):586-600, March 2000.

[8] Anamika Ahirwar and R.S. Jadon, "Tumor Region Extraction in Cancerous Brain MRI Images", published in International Journal of Computer Information Systems(IJCIS ISSN 2229-5208), through Silicon Valley Publishers, Vol 2 No.4, PP 72-95, April 2011.

[9] CBTRUS Statistical Report: Primary Brain and Central Nervous System Tumors Diagnosed in the United States in 2011 . 